\title{
Association between Falls and Nutritional Status of Community-Dwelling Elderly People in Korea
}

\author{
Ah-Ra Jo, Mi-Jeong Park, Byung-Gue Lee, Young-Gyun Seo, Hong-Ji Song, Yu-Jin Paek, Kyung-Hee Park, \\ Hye-Mi Noh* \\ Department of Family Medicine, Hallym University Sacred Heart Hospital, Anyang, Korea
}

\begin{abstract}
Background: Malnutrition is a well-known risk factor of falls, although studies examining the association between nutritional status and falls are rare. We aimed to investigate the association between nutritional status and falls according to gender among Korean older adults.

Methods: The study included 10,675 participants (4,605 men and 6,070 women) aged 65 years and older and used data from the 2011 Survey of Living Conditions and Welfare Needs of Korean Older Persons. Nutritional status of the participants was assessed using the Nutritional Screening Initiative checklist, and the participants were categorized into the following groups: "good," "moderate nutritional risk," and "high nutritional risk." Odds ratios (OR) of fall risk in the above groups based on gender were evaluated using multivariate logistic regression analyses.

Results: Fallers in both genders showed significant association with older age, lower household income, inadequate exercise, and poor nutritional status compared with non-fallers. Considering the good nutritional status group as the reference group, the high nutritional risk group showed a higher risk of falls in men (OR, 1.59; 95\% confidence interval [CI], 1.26-1.99); both moderate and high nutritional risk groups showed a higher risk of falls after adjusting for confounding factors in women (OR, 1.39; 95\% CI, 1.19-1.62 and OR, 1.90; 95\% CI, 1.61-2.24, respectively).

Conclusion: The risk of falls was associated with poor nutritional status, and statistical significance of the association between nutritional status and falls was stronger in women than in men.
\end{abstract}

Keywords: Nutritional Status; Accidental Falls; Aged; Female

Received: July 20, 2018, Revised: January 31, 2019, Accepted: June 9, 2019

*Corresponding Author: Hye-Mi Noh https://orcid.org/0000-0003-2112-1613

Tel: +82-31-380-3805, Fax: +82-31-380-1782, E-mail: hyeminoh@hallym.or.kr 


\section{INTRODUCTION}

Falls are recognized as an important public health challenge for older adults people whose proportion is growing worldwide. ${ }^{1)}$ Every year, $30 \%$ of individuals aged 65 years fall at least once. ${ }^{2)}$ Based on the 2014 Survey of Living Conditions and Welfare Needs of Korean Older Persons, the elderly aged more than 65 years had a $25.1 \%$ fall rate, with an average fall rate of 2.3 times per year. ${ }^{3,4}$ In the United States, fall is the seventh leading cause of death among older adults. ${ }^{5)}$

Many previous studies have reported that the risk of falls is multifactorial. ${ }^{6}$ A number of factors including advancing age, reduced activity, chronic conditions (such as arthritis, neurologic disease, and incontinence), polypharmacy, and age-related changes in gait and balance have been associated with a higher risk of falling. ${ }^{7,8)}$ Inadequate nutritional intake and malnutrition play a cardinal role in over half of the above factors, although studies relating impaired nutritional status to falls are relatively scarce. ${ }^{9,10)}$ Even in healthy individuals, muscle mass declines with age, and muscle function and mass are negatively influenced by malnutrition. ${ }^{11)}$ Furthermore, muscle weakness increases the risk of falling by about fourfold. ${ }^{10)}$

Moreover, despite the majority of studies reporting higher rates of falling in elderly women than in elderly men, studies on gender-specific analyses are rare. ${ }^{7)}$ This gender disparity might be attributed to the significant reduction in bone mineral density and the risk of metabolic syndrome after menopause in women. ${ }^{12)}$

Although the Korean population is rapidly aging, most of the above studies have been conducted predominantly in Western countries, and the Korean national statistics on falls remain sparse, with most of the data obtained from outpatients or nursing home residents and not from community-dwelling elderly. ${ }^{13,14)}$ The present study aimed to investigate the association between nutritional status and falls, gender specifically, based on the 2011 national elderly survey data.

\section{METHODS}

\section{Study Population}

We used the data from the 2011 Survey of Living Conditions and Welfare Needs of Korean Older Persons conducted by the Korea Institute for Health and Social Affairs, between April and November 2011. The quality of the data was evaluated and approved by Statistics Korea of the Korean Government. The survey has been performed at 3-year intervals since 2008 on a representative sample of community-dwelling elderly Korean patients to obtain and assess the baseline data and health indicators necessary for the establishment of policies for the elderly. A stratified, two-stage, cluster sampling design was used to select the representative samples. Face-to-face interviews were conducted by well-trained interviewers who have received training twice or more per year, visited the patients' households for investigation, and recorded the responses in the questionnaire. After excluding elderly people who left the community due to hospitalization, long-term care, or death during the investigation, a total of 10,675 participants aged 65 years and older were included in the final analysis.

All study protocols were approved by the institutional review board (IRB approval no., 2018-04-028). All subjects provided informed consent before participation in the study.

\section{Assessment of Nutritional Status}

The present study used the Nutritional Screening Initiative (NSI) checklist to assess the risk of poor nutritional status or malnutrition in the elderly. This tool was designed in 1991 by the American Academy of Family Physicians, the National Council on the Aging, and the American Dietetic Association to create a coalition with a goal to promote the integration of nutrition screening and intervention into healthcare of older adults. ${ }^{15,16)}$ The checklist included 11 items assessing nine domains: disease, eating poorly, tooth loss/mouth pain, economic hardship, reduced social contact, multiple medicines, involuntary weight loss/gain, needs assistance in self- care, and elderly aged more than 80 years (also known as DETERMINE); we used the Korean version of the above checklist. ${ }^{17)}$ The nutritional status of participants was categorized into three groups and defined per the NSI scores as follows: "good" (0-2 points), "moderate nutritional risk" (3-5 points), and "high nutritional risk" ( $\geq 6$ points).

A review of literature showed that the NSI has been validated in some Western and non-Western populations for identifying older people at risk for malnutrition. ${ }^{18,19)}$

\section{Assessment of Falls}

In the survey, "fall" was defined as a fall, slip, or flop due to any cause irrespective of injury. Those who experienced falls were identified if they responded "yes" to the following question: "In the past 12 months, did you have any falls (including a slip or flop) before the interview?" The survey did not take into account the number of falls.

\section{Measurements of Other Risk Factors}

In addition to nutritional status, we adopted the data from previous studies regarding risk factors for falls collected in the 2011 Survey of Living Conditions and Welfare Needs of Korean Older Persons to evaluate the effects of other potential risk factors on falls including age (65-74 or $\geq 75$ years), gender, body mass index (BMI), education, household income, marital status, smoking, exercise, and comorbidities. BMI was calculated as weight in kilograms divided by the square of height in meters. BMI according to the Clinical Guidelines of Treatment of Obesity conducted by the Korean Society for the Study of Obesity was divided into four categories: low birth weight $\left(<18.5 \mathrm{~kg} / \mathrm{m}^{2}\right)$, normal weight (18.5-22.9 kg/m²), overweight $\left(23.0-24.9 \mathrm{~kg} / \mathrm{m}^{2}\right)$, and obesity $\left(\geq 25.0 \mathrm{~kg} / \mathrm{m}^{2}\right)$. Education was categorized into less than middle school and post-middle school. Marital status was divided into two categories (married and single/widowed/divorced). Smoking status was classified as never-smoker, former smoker, and current smoker. Exercise was categorized as no/inadequate versus adequate exercise defined as more than 150 minutes per week. History of hypertension, diabetes mellitus, stroke, arthritis, eye disorders (including cataracts 
and/or glaucoma), osteoporosis, chronic otitis media, heart disease, urinary incontinence, anemia, and depression were considered covariates. These were defined as "long-term conditions that are expected to last or have already lasted 3 months or more and that have been diagnosed by a health professional." Each condition was assessed as a binary variable (yes versus no).

\section{Statistical Analysis}

Descriptive statistics were used to summarize the participants' characteristics. To account for the complex sampling design of the 2011 Survey of Living Conditions and Welfare Needs of Korean Older Persons including nonresponses, we used sampling weights in all the analyses. The chi-square test was used to compare the differences between nonfallers and fallers among the older adults aged more than 65 years in terms of gender, age, education, household income, marital status, smoking, exercise, and comorbidities.

Multivariate logistic regression analyses were performed to assess the relationships between nutritional status and fallers; we ran four models that included an increasing number of covariates and had different standards depending on gender based on the results of the chisquare tests. In men aged 65 years or older, model 1 (minimally adjusted) included age; model 2 was adjusted as in model 1 but also included education and household income; model 3 was adjusted as in model 2 but also included exercise and BMI; model 4 (fully adjusted) was adjusted as in model 3 but also included comorbidities (hypertension, diabetes mellitus, stroke, arthritis, eye disorders such as cataracts and/or glaucoma, osteoporosis, heart disease, anemia, and depression). In women aged 65 years or older, model 1 (minimally adjusted) included age; model 2 was adjusted as in model 1 but also included household income and marital status; model 3 was adjusted as in model 2 but also included smoking, exercise and BMI; model 4 (fully adjusted) was adjusted as in model 3 but also included comorbidities (hypertension, diabetes mellitus, stroke, arthritis, eye disorders such as cataracts and/or glaucoma, osteoporosis, chronic otitis media, urinary incontinence, anemia, and depression).

Linear regression analyses were adopted to calculate the $\mathrm{P}$ for trend. Linear regression analyses for the $\mathrm{P}$ for trends and logistic regression analyses for the odds ratios (ORs) were also used. In all analyses, we used 95\% confidence intervals (95\% CI); two-tailed P-values $<0.05$ were considered significant. Data were analyzed using the software IBM SPSS ver. 20.0 (IBM Corp., Armonk, NY, USA).

\section{RESULTS}

Figures 1 and 2 show the percentage of participants who experienced falls according to nutritional status in both genders, respectively. The high nutritional risk group showed higher percentage of fallers than non-fallers in both sexes. Furthermore, the good nutritional status group showed higher percentage of non-fallers than fallers in both sexes.

Table 1 shows the general characteristics of the study participants. Fall prevalence increased with age. The prevalence of falls was $14.8 \%$ in men and $25.9 \%$ in women. Compared with non-fallers, fallers had a significantly higher proportion of those with obesity, an education level less than middle school, a low household income, a single/widowed/divorced marital status, and no/inadequate exercise. Furthermore, a higher proportion of fallers reported poor nutritional status than non-fallers. However, non-fallers had a higher rate of a normal weight and current smoking. Compared with non-fallers, a higher percentage of fallers had hypertension, diabetes mellitus, stroke, arthritis, eye disorders (including cataracts and/or glaucoma), osteoporosis, chronic otitis media, heart disease, urinary incontinence, anemia, and depression.

Table 2 shows the sociodemographic and lifestyle/behavioral corre-

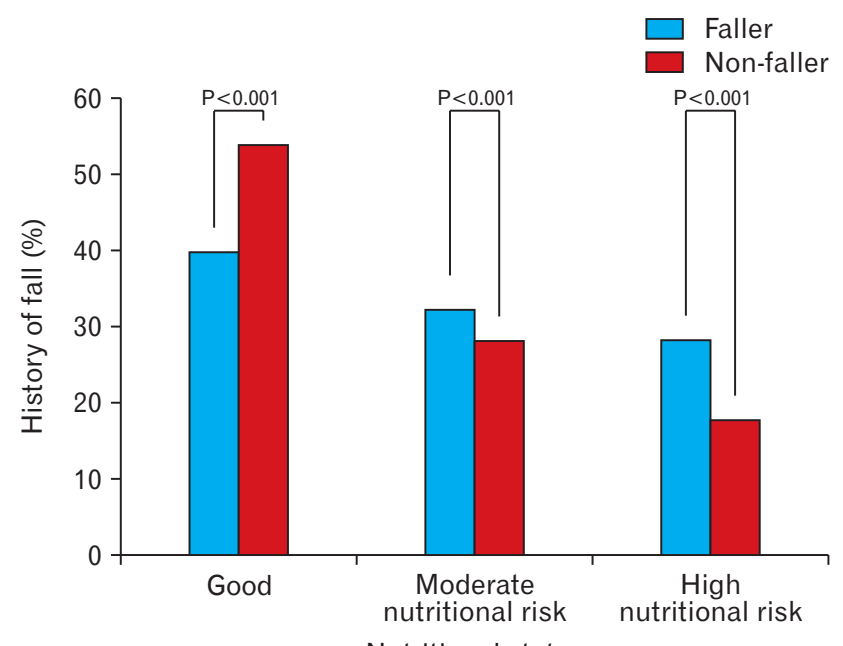

Figure 1. Percentage of history of fall according to nutritional status in men. P-values were calculated using chi-square tests to compare the differences between groups.

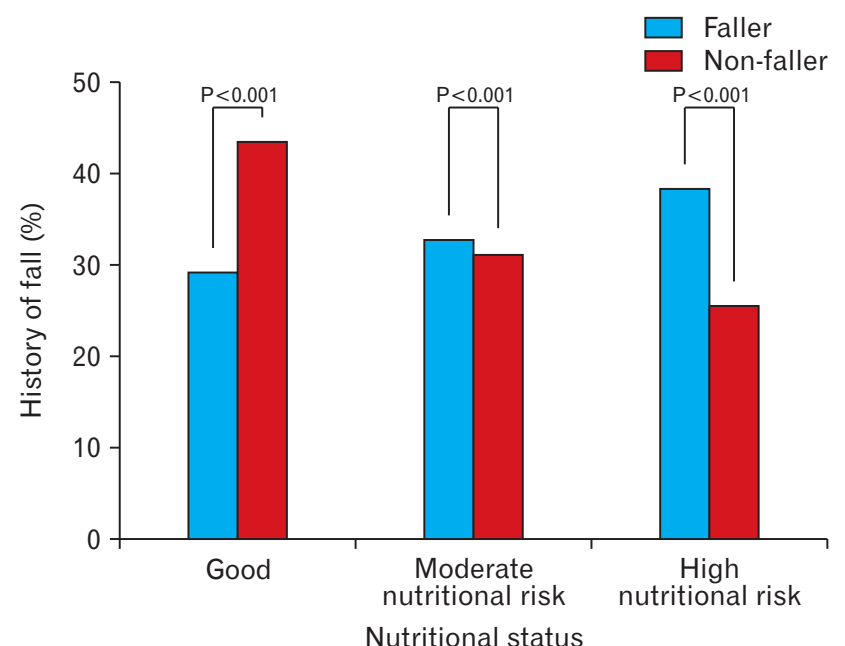

Figure 2. Percentage of history of fall according to nutritional status in women. P-values were calculated using chi-square tests to compare the differences between groups. 
Table 1. General characteristics of the study participants based on the prevalence of falls

\begin{tabular}{|c|c|c|c|c|}
\hline \multirow{2}{*}{ Characteristic } & \multirow{2}{*}{ Total $(n=10,675)$} & \multicolumn{2}{|c|}{ Falls } & \multirow{2}{*}{ P-value* } \\
\hline & & Yes $(n=2,258)$ & No $(n=8,417)$ & \\
\hline Age $(y)$ & & & & $<0.001$ \\
\hline $65-74$ & $6,420(60.1)$ & $1,193(52.8)$ & $5,227(62.1)$ & \\
\hline$\geq 75$ & $4,255(39.9)$ & $1,065(47.2)$ & $3,190(37.9)$ & \\
\hline Male sex & $4,605(43.1)$ & $682(30.2)$ & $3,923(46.6)$ & $<0.001$ \\
\hline Body mass index $\left(\mathrm{kg} / \mathrm{m}^{2}\right)$ & & & & 0.001 \\
\hline Underweight $(<18.5)$ & $568(5.5)$ & $129(5.8)$ & $439(5.4)$ & \\
\hline Normal weight (18.5-22.9) & $4,268(41.0)$ & $875(39.6)$ & $3,393(41.4)$ & \\
\hline Overweight (23.0-24.9) & $2,536(24.4)$ & $490(22.2)$ & $2,046(25.0)$ & \\
\hline Obesity $(\geq 25.0)$ & $3,037(29.2)$ & $715(32.4)$ & $2,322(28.3)$ & \\
\hline Education & & & & $<0.001$ \\
\hline Less than middle school & $7,164(67.1)$ & $1,705(75.5)$ & $5,459(64.9)$ & \\
\hline Post-middle school & $3,511(32.9)$ & $553(24.5)$ & $2,958(35.1)$ & \\
\hline Household income (10,000 Korean won) & & & & $<0.001$ \\
\hline 1 Quintile & $2,135(20.0)$ & $575(25.5)$ & $1,560(18.5)$ & \\
\hline 2 Quintile & $2,138(20.0)$ & $434(19.2)$ & $1,704(20.2)$ & \\
\hline 3 Quintile & $2,132(20.0)$ & $443(19.6)$ & $1,689(20.1)$ & \\
\hline 4 Quintile & $2,135(20.0)$ & $432(19.1)$ & $1,703(20.2)$ & \\
\hline 5 Quintile & $2,134(20.0)$ & $374(16.6)$ & $1,760(20.9)$ & \\
\hline Marital status & & & & $<0.001$ \\
\hline Single/widowed/divorced & $3,475(32.6)$ & 930 (41.2) & 2,545 (30.2) & \\
\hline Married & $7,199(67.4)$ & $1,327(58.8)$ & $5,872(69.8)$ & \\
\hline Smoking status & & & & $<0.001$ \\
\hline Never smoker & $6,567(61.5)$ & $1,559(69.0)$ & $5,008(59.5)$ & \\
\hline Former smoker & $2,781(26.1)$ & $457(20.2)$ & $2,324(27.6)$ & \\
\hline Current smoker & $1,327(12.4)$ & $242(10.7)$ & $1,085(12.9)$ & \\
\hline Exercise & & & & $<0.001$ \\
\hline No/inadequate & $6,442(60.4)$ & $1,499(66.4)$ & $4,943(58.7)$ & \\
\hline Adequate exercise & $4,231(39.6)$ & $758(33.6)$ & $3,473(41.3)$ & \\
\hline Nutritional status (score) & & & & $<0.001$ \\
\hline Good & $4,791(45.0)$ & $730(32.4)$ & $4,061(48.4)$ & \\
\hline Moderate nutritional risk & $3,233(30.4)$ & $733(32.5)$ & $2,500(29.8)$ & \\
\hline High nutritional risk & $2,625(24.7)$ & $792(35.1)$ & $1,833(21.8)$ & \\
\hline \multicolumn{5}{|l|}{ Comorbidities } \\
\hline Hypertension & $5,852(54.8)$ & $1,348(59.7)$ & $4,504(53.5)$ & $<0.001$ \\
\hline Diabetes mellitus & $2,174(20.4)$ & $563(24.9)$ & $1,611(19.1)$ & $<0.001$ \\
\hline Stroke & $824(7.7)$ & $246(10.9)$ & $578(6.9)$ & $<0.001$ \\
\hline Arthritis & 3,976 (37.2) & $1,083(48.0)$ & 2,893 (34.4) & $<0.001$ \\
\hline Eye disorder & $1,225(11.5)$ & 337 (14.9) & $888(10.6)$ & $<0.001$ \\
\hline Osteoporosis & $1,797(16.8)$ & $546(24.2)$ & $1,251(14.9)$ & $<0.001$ \\
\hline Chronic otitis media & $78(0.7)$ & $28(1.2)$ & $50(0.6)$ & 0.001 \\
\hline Heart disease & $1,215(11.4)$ & $291(12.9)$ & $924(11.0)$ & 0.011 \\
\hline Urinary incontinence & $227(2.1)$ & $84(3.7)$ & $143(1.7)$ & $<0.001$ \\
\hline Anemia & $275(2.6)$ & $103(4.6)$ & $172(2.0)$ & $<0.001$ \\
\hline Depression & $327(3.1)$ & $106(4.7)$ & $221(2.6)$ & $<0.001$ \\
\hline
\end{tabular}

Values are presented as number (\%) for categorical variables.

*Obtained using chi-square test.

lates of falls based on gender. Fall prevalence increased with age in both genders. An older age, a low household income, no/inadequate exercise, poor nutritional status, and comorbidities (excluding chronic otitis media and heart disease) were significantly associated with falls in both genders; a normal weight, an education level less than middle school, and heart disease were significantly associated with falls in men; obesity, being single/widowed/divorced, former/current smok- ing, and chronic otitis media were significantly associated with falls in women.

Tables 3 and 4 show the results of multivariate logistic regression analysis of the data on nutritional status and falls in men and women, respectively. Among men, as shown in Table 3, a poorer nutritional status was associated with a higher risk of falls in model 1 . The associations were similar after adjustment for education and household in- 
Table 2. General characteristics of the study participants based on the prevalence of falls by gender

\begin{tabular}{|c|c|c|c|c|c|c|}
\hline \multirow{3}{*}{ Characteristic } & \multicolumn{6}{|c|}{ Falls } \\
\hline & \multicolumn{3}{|c|}{ Men } & \multicolumn{3}{|c|}{ Women } \\
\hline & Yes $(n=682)$ & No $(n=3,923)$ & P-value* & Yes $(n=1,577)$ & No $(n=4,493)$ & P-value* \\
\hline Age (y) & & & $<0.001$ & & & $<0.001$ \\
\hline $65-74$ & $379(55.6)$ & $2,579(65.7)$ & & $814(51.6)$ & $2,647(58.9)$ & \\
\hline$\geq 75$ & $303(44.4)$ & $1,344(34.3)$ & & $763(48.4)$ & $1,846(41.1)$ & \\
\hline Body mass index $\left(\mathrm{kg} / \mathrm{m}^{2}\right)$ & & & 0.032 & & & 0.019 \\
\hline Underweight (<18.5) & $48(7.2)$ & $240(6.3)$ & & $81(5.3)$ & $199(4.5)$ & \\
\hline Normal weight (18.5-22.9) & $326(48.7)$ & $1,673(43.7)$ & & $549(35.6)$ & $1,720(39.3)$ & \\
\hline Overweight (23.0-24.9) & $155(23.2)$ & $1,062(27.8)$ & & $335(21.8)$ & $983(22.5)$ & \\
\hline Obesity $(\geq 25.0)$ & $140(20.9)$ & $850(22.2)$ & & $575(37.3)$ & $1,472(33.7)$ & \\
\hline Education & & & $<0.001$ & & & 0.050 \\
\hline Less than middle school & $371(54.5)$ & $1,752(44.7)$ & & $1,334(84.6)$ & $3,707(82.5)$ & \\
\hline Post-middle school & $310(45.5)$ & $2,171(55.3)$ & & $242(15.4)$ & $787(17.5)$ & \\
\hline Household income (10,000 Korean won) & & & $<0.001$ & & & 0.044 \\
\hline 1 Quintile & $123(18.1)$ & $422(10.8)$ & & $452(28.7)$ & $1,138(25.3)$ & \\
\hline 2 Quintile & $122(17.9)$ & $804(20.5)$ & & $312(19.8)$ & $900(20.0)$ & \\
\hline 3 Quintile & $167(24.5)$ & $905(23.1)$ & & $275(17.4)$ & $784(17.4)$ & \\
\hline 4 Quintile & $152(22.3)$ & $886(22.6)$ & & $280(17.8)$ & $817(18.2)$ & \\
\hline 5 Quintile & $117(17.2)$ & $907(23.1)$ & & $257(16.3)$ & $854(19.0)$ & \\
\hline Marital status & & & 0.240 & & & $<0.001$ \\
\hline Single/widowed/divorced & $72(10.6)$ & $359(9.2)$ & & $858(54.4)$ & $2,185(48.6)$ & \\
\hline Married & $609(89.4)$ & $3,564(90.8)$ & & $718(45.6)$ & $2,308(51.4)$ & \\
\hline Smoking status & & & 0.716 & & & $<0.001$ \\
\hline Never smoker & $135(19.8)$ & $816(20.8)$ & & $1,423(90.3)$ & $4,192(93.3)$ & \\
\hline Former smoker & $372(54.6)$ & $2,155(54.9)$ & & $85(5.4)$ & $168(3.7)$ & \\
\hline Current smoker & $174(25.6)$ & $952(24.3)$ & & $68(4.3)$ & $133(3.0)$ & \\
\hline Exercise & & & 0.011 & & & $<0.001$ \\
\hline No/inadequate & $382(56.1)$ & $1,994(50.8)$ & & $1,117(70.9)$ & $2,950(65.7)$ & \\
\hline Adequate exercise & $299(43.9)$ & $1,930(49.2)$ & & $459(29.1)$ & $1,543(34.3)$ & \\
\hline Nutritional status (score) & & & $<0.001$ & & & $<0.001$ \\
\hline Good & $271(39.7)$ & $2,111(54.0)$ & & 459 (29.2) & $1,950(43.5)$ & \\
\hline Moderate nutritional risk & 219 (32.1) & $1,108(28.3)$ & & $514(32.7)$ & $1,392(31.0)$ & \\
\hline High nutritional risk & $192(28.2)$ & $690(17.7)$ & & $600(38.1)$ & $1,144(25.5)$ & \\
\hline \multicolumn{7}{|l|}{ Comorbidities } \\
\hline Hypertension & 363 (53.3) & $1,888(48.1)$ & 0.012 & $985(62.5)$ & $2,617(58.2)$ & 0.003 \\
\hline Diabetes mellitus & $174(25.6)$ & 717 (18.3) & $<0.001$ & $389(24.7)$ & 893 (19.9) & $<0.001$ \\
\hline Stroke & $115(16.9)$ & $313(8.0)$ & $<0.001$ & $131(8.3)$ & 265 (5.9) & 0.001 \\
\hline Arthritis & $169(24.8)$ & $663(16.9)$ & $<0.001$ & $914(58.0)$ & $2,230(49.6)$ & $<0.001$ \\
\hline Eye disorder & 85 (12.5) & $311(7.9)$ & $<0.001$ & $252(16.0)$ & $576(12.8)$ & 0.002 \\
\hline Osteoporosis & $40(5.9)$ & $125(3.2)$ & $<0.001$ & $506(32.1)$ & $1,126(25.1)$ & $<0.001$ \\
\hline Chronic otitis media & $4(0.6)$ & $13(0.3)$ & 0.310 & $25(1.6)$ & $38(0.8)$ & 0.013 \\
\hline Heart disease & $95(14.0)$ & $424(10.8)$ & 0.017 & 196 (12.4) & $500(11.1)$ & 0.161 \\
\hline Urinary incontinence & $1(0.1)$ & $7(0.2)$ & 0.855 & $83(5.3)$ & $136(3.0)$ & $<0.001$ \\
\hline Anemia & $16(2.3)$ & $49(1.2)$ & 0.025 & $87(5.5)$ & $123(2.7)$ & $<0.001$ \\
\hline Depression & $21(3.1)$ & $57(1.5)$ & 0.002 & $84(5.3)$ & $164(3.7)$ & 0.004 \\
\hline
\end{tabular}

Values are presented as number (\%) for categorical variables.

${ }^{*}$ Obtained using chi-square test.

come in model 2; after further adjustment, the magnitude of associations was slightly attenuated in models 3 and 4 . In men, the OR of the high nutritional risk group showed a higher risk compared with that in the reference group in model 4 (OR, 1.59; 95\% CI, 1.26-1.99). However, the risk of falls in the moderate nutritional risk group lost significance (OR, 1.21; 95\% CI, 0.98-1.49) in model 4, which was further adjusted for comorbidities. The associations in the moderate nutritional risk group did not reach statistical significance, but men tended to show an increased risk of falls based on their nutritional status ( $\mathrm{P}$ for the trend $<0.001)$.

Among women, as shown in Table 4, a poorer nutritional status was associated with a higher risk of falls in model 1 . The associations were similar after adjustment for household income and marital status in model 2; after further adjustment, the magnitude of associations was 
Table 3. Multivariate logistic regression analysis of nutritional status and prevalence of falls in men aged 65 years or older

\begin{tabular}{lcccc}
\hline & Model 1 & Model 2 & Model 3 & Model 4 \\
\hline Nutritional status & & & & \\
$\quad$ Good $(\mathrm{n}=2,382)$ & Reference & Reference & Reference & Reference \\
$\quad$ Moderate nutritional risk $(\mathrm{n}=1,327)$ & $1.5(1.23-1.82)$ & $1.46(1.20-1.77)$ & $1.50(1.23-1.83)$ & $1.21(0.98-1.49)$ \\
$\quad$ High nutritional risk $(\mathrm{n}=882)$ & $2.07(1.68-2.54)$ & $1.94(1.56-2.40)$ & $1.96(1.57-2.44)$ & $1.59(1.26-1.99)$ \\
P for trend & & & & $<0.001$ \\
\hline
\end{tabular}

Values are presented as odds ratio (95\% confidence interval). Model 1: adjusted for age; model 2: adjusted for age, education, and household income; model 3: adjusted for age, education, household income, exercise, and body mass index; model 4: adjusted for age, education, household income, exercise, body mass index, and comorbidities (hypertension, stroke, heart disease, diabetes mellitus, arthritis, osteoporosis, eye disorders, anemia, and depression).

*P for trend was determined by linear regression analysis.

Table 4. Multivariate logistic regression analysis of nutritional status and prevalence of falls in women aged 65 years or older

\begin{tabular}{lcccc}
\hline & Model 1 & Model 2 & Model 3 & Model 4 \\
\hline Nutritional status & & & & Reference \\
Good $(\mathrm{n}=2,409)$ & Reference & Reference & Reference & $1.39(1.19-1.62)$ \\
$\quad$ Moderate nutritional risk $(\mathrm{n}=1,906)$ & $1.53(1.33-1.77)$ & $1.55(1.34-1.80)$ & $1.52(1.31-1.77)$ & $1.90(1.61-2.24)$ \\
$\quad$ High nutritional risk $(\mathrm{n}=1,744)$ & $2.16(1.87-2.49)$ & $2.22(1.91-2.59)$ & $2.17(1.85-2.54)$ & $<0.001$ \\
P for trend* & & & &
\end{tabular}

Values are presented as odds ratio (95\% confidence interval). Model 1: adjusted for age; model 2: adjusted for age, household income, and marital status; model 3: adjusted for age, household income, marital status, smoking, exercise, and body mass index; model 4: adjusted for age, household income, marital status, smoking, exercise, body mass index, and comorbidities (hypertension, stroke, diabetes mellitus, arthritis, osteoporosis, eye disorders, chronic otitis media, urinary incontinence, anemia, and depression).

${ }^{*} \mathrm{P}$ for trend was determined by linear regression analysis.

slightly attenuated in models 3 and 4 . Model 4 showed a higher rate of falls in both moderate and high nutritional risk groups (OR, 1.39; 95\% CI, 1.19-1.62; $\mathrm{P}<0.001$ and OR, 1.90; 95\% CI, 1.61-2.24; $\mathrm{P}<0.001$, respectively). Women also tended to show an increased risk of falls based on their nutritional status ( $\mathrm{P}$ for the trend $<0.001$ ).

\section{DISCUSSION}

In this study of 10,675 participants aged 65 years and older, we found that the risk of falls was more significantly associated with poor nutritional status than with good nutritional status. In addition, we found that the statistical significance of the association between nutritional status and falls was stronger in women than in men. Especially in women, the moderate nutritional risk and the high nutritional risk group showed significantly higher ORs for falls than the good nutritional status group, after correction of factors including household income, marital status, and smoking.

Although studies on this subject are limited, and most studies have focused on the different factors affecting falls in Korea, some studies have also identified that malnutrition is a risk factor for falls in elderly individuals. Our results were consistent with those of two earlier studies, in particular those published by Chien and $\mathrm{Guo}^{17}$ in Taiwan and by Neyens et al. ${ }^{10)}$ in the Netherlands. A study in Taiwan found an association between baseline nutritional status and incidence of falls among 4,440 community-dwelling Taiwanese aged 53 years and older. ${ }^{17)}$ Moreover, a study in the Netherlands found that malnutrition was associated with an increased risk of falls, which was supported by the fact that there was a positive effect of nutritional intervention on the risk of falls. ${ }^{10)}$

The relationship between falls and nutritional status in the current study may be explained by the frailty process. Frailty may be caused by a disease, lack of activity, inadequate nutritional intake, multiple hospitalizations, poor dentition, and/or the physiologic changes associated with aging such as decreased metabolic rate. ${ }^{20)}$ These multiple causes can lead to chronic malnutrition and sarcopenia, which may cause decreased strength and impaired balance, ultimately leading to falls. ${ }^{21)}$ It is meaningful that the questions of the NSI checklist used in this study are consistent with the causes of frailty.

There are important dietary components that may help explain the link between falls and nutritional status. First, the chronic intake of excess acid-producing nutrients such as meat and cereal grains in combination with a low intake of the alkalinizing fruits and vegetables may lead to a chronic acid challenge and stimulate muscle catabolism, causing an increased risk of falls. ${ }^{6}$ Second, for each $1 \mathrm{ng} / \mathrm{mL}$ increase in serum folate, the risk for falls decreases by $19 \%$. The mechanism underlying the negative association between folate and falls is still obscure, but it may be related to the folate's contribution to adequate cell division or to the toxic effect of elevated homocysteine levels stemming from lower folate levels. ${ }^{9)}$ A study in Japan also showed that vita$\min B_{12}$ and/or folic acid might improve postural stability and/or muscle function and strength, thus helping to reduce falls. ${ }^{22)}$ Third, low serum vitamin $\mathrm{D}$ (serum 25-hydroxyvitamin $\mathrm{D}<25 \mathrm{nmol} / \mathrm{L}$ ) and calcium levels have been associated with muscle weakness, poor physical performance, balance problems, and falls in older adults. ${ }^{23)}$ A possible 
mechanistic explanation is that vitamin D plays an important role in muscle function through its regulation of calcium transport and protein synthesis in the muscle. In addition, vitamin D deficiency has been recognized as a cause of secondary hyperparathyroidism. Moreover, increase in parathyroid hormone (PTH) levels is associated with increased protein catabolism and decrease in the number of type 2 muscle fibers in animal models. PTH also induces the production of interleukin 6, which is associated with lower muscle strength ${ }^{24)}$ and poor physical performance. ${ }^{6)}$ Finally, people with malnutrition also tend to have nutrition-related health problems, which are additional risk factors for falls in the elderly. ${ }^{17)}$ Thus, our study provides further evidence of the increased propensity to fall due to malnutrition.

In addition to nutritional status, our results confirm that older women (OR, 1.90; 95\% CI, 1.61-2.24; $\mathrm{P}<0.001)$ were more likely to fall than older men (OR, 1.59; 95\% CI, 1.26-1.99; $\mathrm{P}<0.001$ ). The underlying mechanisms linking gender and falling may involve gender-based differences in psychosocial aspects and bone and muscle balance due to aging, as well as hormonal changes associated with menopause. A previous gender-based study using a fall prediction model of community-dwelling elderly in Korea found that the fear of falling directly affects the incidence of falls among female older adults, increases with age, is higher in women, and negatively affects activity level. As a result, the fear of falling increases the risk of falls. ${ }^{25,26)}$ Furthermore, a study on fall risk-related factors in postmenopausal women in Korea found that in postmenopausal women, bone loss is predicted because of the shortage of estrogen, resulting in rapid bone resorption. In addition, the prevalence of metabolic syndrome is rapidly increased after menopause, and the ability to control one's body such as a sense of balance is reduced. ${ }^{12)}$ Therefore, to effectively prevent falls among the elderly in the community, a method for assessing the gender-dependent risk of falling should be developed.

Our study had three limitations. First, owing to its cross-sectional study design, we could not establish the causal relationship between nutritional status and falling. Second, we relied on self-reported data of the incidence of falls, which might have been underestimated because some older individuals might not have recalled all the falls. Finally, our study investigated the number of drugs taken, but did not determine the types of drugs, some of which are known to affect falls such as psychotropics, antidiabetics, and nonsteroidal anti-inflammatory drugs. Therefore, the possible effects of drugs on the risk of falls were not considered.

Despite these limitations, to the best of our knowledge, the present study is the first to investigate the relationship between nutritional status and falls based on gender among Korean elderly. Moreover, we randomly sampled the community-dwelling elderly, not hospitals or nursing homes, to assess real-life fall incidence. Data were obtained using a direct interview method, and previous medical history including physician diagnosis supported the objectivity of the study. In conclusion, the present study demonstrates that nutritional status, especially that assessed using the NSI checklist, is significantly inversely associated with the risk of falls among Korean elderly, and the statistical significance of the association between nutritional status and falls is stronger in women than in men. Further studies are needed to identify the types of nutritional interventions that can help prevent falls in the elderly based on gender.

\section{CONFLICT OF INTEREST}

No potential conflict of interest relevant to this article was reported.

\section{ORCID}

Ah-Ra Jo: https://orcid.org/0000-0003-4196-1273

Mi-Jeong Park: https://orcid.org/0000-0003-1664-4452

Byung-Gue Lee: https://orcid.org/0000-0001-6432-2453

Young-Gyun Seo: https://orcid.org/0000-0001-8294-1741

Hong-Ji Song: https://orcid.org/0000-0002-3563-9504

Yu-Jin Paek: https://orcid.org/0000-0001-9573-8849

Kyung-Hee Park: https://orcid.org/0000-0001-9806-0076

Hye-Mi Noh: https://orcid.org/0000-0003-2112-1613

\section{REFERENCES}

1. Torres MJ, Feart C, Samieri C, Dorigny B, Luiking Y, Berr C, et al. Poor nutritional status is associated with a higher risk of falling and fracture in elderly people living at home in France: the three-city cohort study. Osteoporos Int 2015;26:2157-64.

2. Tinetti ME, Kumar C. The patient who falls: "it's always a trade-off". JAMA 2010;303:258-66.

3. Hwang YS, Oh BT, Lee JY, Park SK, Hong SW, Kim DH, et al. Factors related with fear of falling in the Korean elderly. Korean J Fam Pract 2017;7:640-5.

4. Korea Institute for Health and Social Affairs. The 2014 survey of the elderly. Sejong: Korea Institute for Health and Social Affairs; 2014.

5. Burns E, Kakara R. Deaths from falls among persons aged $\geq 65$ years: United States, 2007-2016. MMWR Morb Mortal Wkly Rep 2018;67:50914.

6. Ravi N. Association of overall diet quality with falls and physical function among community dwelling older adults-results from MrOS study [master's thesis]. Pittsburgh (PA): University of Pittsburgh; 2014.

7. Chang VC, Do MT. Risk factors for falls among seniors: implications of gender. Am J Epidemiol 2015;181:521-31.

8. Ambrose AF, Paul G, Hausdorff JM. Risk factors for falls among older adults: a review of the literature. Maturitas 2013;75:51-61.

9. Shahar D, Levi M, Kurtz I, Shany S, Zvili I, Mualleme E, et al. Nutritional status in relation to balance and falls in the elderly: a preliminary look at serum folate. Ann Nutr Metab 2009;54:59-66.

10. Neyens J, Halfens R, Spreeuwenberg M, Meijers J, Luiking Y, Verlaan G, et al. Malnutrition is associated with an increased risk of falls and impaired activity in elderly patients in Dutch residential long-term care (LTC): a cross-sectional study. Arch Gerontol Geriatr 2013;56:265-9.

11. Vivanti A, Ward N, Haines T. Nutritional status and associations with falls, balance, mobility and functionality during hospital admission. J Nutr Health Aging 2011;15:388-91. 
12. Lee JH, Kim HS. Fall risk related factors in postmenopausal women. Korean J Adult Nurs 2014;26:533-54.

13. Park K, Eun SJ, Lee EJ, Lee CE, Park DY, Han K, et al. The incidence and patterns of unintentional injuries in daily life in Korea: a nationwide study. J Prev Med Public Health 2008;41:265-71.

14. Kim KI, Jung HK, Kim CO, Kim SK, Cho HH, Kim DY, et al. Evidencebased guidelines for fall prevention in Korea. J Korean Geriatr Soc 2016;20:1-28.

15. Yang S. Factors influencing depression of elderly women in a metropolitan city. J Korean Public Health Nurs 2012;26:158-73.

16. Kim MH, Chung HK. Relationship between sense of belonging, powerlessness and nutritional status of elderly people. J Korean Soc Food Cult 2015;30:118-28.

17. Chien MH, Guo HR. Nutritional status and falls in community-dwelling older people: a longitudinal study of a population-based random sample. PLoS One 2014;9:e91044.

18. Moon HK, Kong JE. Reliability of nutritional screening using DETERMINE checklist for elderly in Korean rural areas by season. Korean J Community Nutr 2009;14:340-53.

19. Posner BM, Jette AM, Smith KW, Miller DR. Nutrition and health risks in the elderly: the nutrition screening initiative. Am J Public Health
1993;83:972-8.

20. Ahmed N, Mandel R, Fain MJ. Frailty: an emerging geriatric syndrome. Am J Med 2007;120:748-53.

21. Woo N, Kim SH. Sarcopenia influences fall-related injuries in community-dwelling older adults. Geriatr Nurs 2014;35:279-82.

22. Sato Y, Honda Y, Iwamoto J, Kanoko T, Satoh K. Effect of folate and mecobalamin on hip fractures in patients with stroke: a randomized controlled trial. JAMA 2005;293:1082-8.

23. Houston DK, Cesari M, Ferrucci L, Cherubini A, Maggio D, Bartali B, et al. Association between vitamin $\mathrm{D}$ status and physical performance: the InCHIANTI study. J Gerontol A Biol Sci Med Sci 2007;62:440-6.

24. Visser M, Pahor M, Taaffe DR, Goodpaster BH, Simonsick EM, Newman $\mathrm{AB}$, et al. Relationship of interleukin- 6 and tumor necrosis factoralpha with muscle mass and muscle strength in elderly men and women: the Health ABC Study. J Gerontol A Biol Sci Med Sci 2002;57:M326-32.

25. Yun ES. Fall prediction model for community-dwelling elders based on gender. J Korean Acad Nurs. 2012 Dec;42(6):810-8.

26. Lim JY, Park WB, Oh MK, Kang EK, Paik NJ. Falls in a proportional region population in Korean elderly: incidence, consequences, and risk factors. J Korean Geriatr Soc 2010;14:8-17. 